\title{
Crecimiento endógeno en un país menos desarrollado: el caso de firmas imitadoras
}

\author{
Raymundo Vite Cristóbal*
}

\section{RESUMEN}

El tema del crecimiento endógeno en un país menos desarrollado no es tan común en la nueva bibliografía sobre crecimiento económico; la mayor parte del esfuerzo teórico se ha centrado en el análisis de países desarrollados. En este artículo, inspirado en Romer (1990), Romer y Rivera-Batiz (1991) y Tirado (1995), se expone un modelo de crecimiento endógeno para un país menos desarrollado en donde la fuerza que impulsa el crecimiento económico es la imitación tecnológica de diseños de bienes de capital innovadores. El modelo muestra evidencia analítica de que la tasa de crecimiento de un país menos desarrollado mantiene una relación creciente con la brecha tecnológica y el acervo de capital humano total de la economía, y una relación decreciente con la tasa de descuento.

Número de clasificación: JEL: O10 O30 O40.

Palabras clave: innovación tecnológica, imitación tecnológica, crecimiento económico, capital humano y brecha tecnológica.

\section{Abstract}

The issue about the endogenous growth at developed country less is not so common in the new literature of economic growth, the majority of the theoretical effort has concentrated in the analysis about developed countries. At this article, inspired by Romer (1990), Romer and Rivera-Batiz (1991) and Tirado (1995), is exposed a model about endogenous growth for a developed country less where the force that impels the economic growth is the technological imitation of designs innovating capital goods. The model shows to analytical evidence where the growth rate in a developed country less maintains increasing relation with the technological gap and the stock of human capital total in the economy, and a decreasing relation with the discount rate.

Number of classificatión: JEL: O10 O30 O40.

Key words: technological innovation, technological imitation, economic growth, capital human and technological gap.

\footnotetext{
* Profesor titular de la licenciatura en economía en la Universidad Autónoma MetropolitanaXochimilco, y en la Escuela Nacional de Estudios Profesionales-Acatlán, de la UNAM.
} 


\section{INTRODUCCIÓN}

El modelo que se presenta se ubica en el marco de la bibliografía sobre crecimiento endógeno de economías abiertas, específicamente en la línea de investigación desarrollada en los trabajos pioneros de Grossman y Helpman (1990 y 1991), Romer y Rivera-Batiz (1991) y Aghion y Howitt (1998), en donde se analizan los efectos del comercio internacional sobre la tasa de crecimiento de los países.

La nueva bibliografía sobre crecimiento económico para economías abiertas se ha centrado en formulaciones de crecimiento endógeno en un entorno de países desarrollados, y muy poco entre economías con distintos niveles de desarrollo, excepto Grossman y Helpman (1991), Young (1991), Tirado (1995) y Aghion y Howitt (1998). La forma en la cual se procede en estos modelos es estableciendo primero las hipótesis de generación de crecimiento endógeno para cada país y después analizando los efectos del comercio internacional sobre la tasa de crecimiento de largo plazo de cada país.

Tirado (1995), inspirado en Romer (1990) y Romer y Rivera-Batiz (1991), presenta un modelo de dos países con actividades de innovación e imitación plenamente diferenciadas: el país innovador produce diseños de bienes de demanda final y el país imitador adapta estos diseños innovadores a las necesidades del mercado y a las características del aparato productivo local. Haciendo abstracción del comercio internacional y del estudio de las ventajas comparativas, se sostiene que la tasa de crecimiento del país imitador depende fundamentalmente de la mano de obra utilizada en el sector de investigación y desarrollo y de la brecha tecnológica entre el país innovador y el país imitador.

Este último resultado refleja las condiciones del país imitador, en gran medida comparable al hallazgo de Grossman y Helpman (1991), en donde la actividad de imitación depende de la razón entre salarios del país imitador y salarios del país innovador.

La propuesta de Tirado (1991), respecto a los modelos de base de Romer (1990) y Romer y Rivera-Batiz (1991), tiene el inconveniente de perder el lado de la demanda final. En el esfuerzo analítico que se presenta en este trabajo se asume que el país imitador se orienta a la adaptación de bienes de capital innovadores en lugar de bienes finales innovadores; la ventaja de la propuesta es que se recupera el lado de la demanda final. Desde este punto de vista, el modelo que ahora se expone puede verse como una adaptación de la propuesta de Tirado (1991). 
Una particularidad de la nueva bibliografía es que la producción y el flujo de conocimiento nuevo entre sectores y entre países tienen la cualidad de generar rendimientos crecientes a escala y así incentivar el crecimiento económico. En el modelo propuesto, al igual que Grossman y Helpman (1991), Aghion y Howitt (1998) y Tirado (1995), la actividad de imitación tecnológica también posee la cualidad de generar rendimientos crecientes a escala y así impulsar el crecimiento económico de largo plazo. Esto nos muestra que dicha actividad también responde a los incentivos del mercado que, al igual que la innovación tecnológica, requiere de asignación de recursos financieros para su creación y utilización.

El objetivo del trabajo es exponer un modelo de crecimiento endógeno para el caso de un país menos desarrollado, en donde el avance económico descanse en la capacidad de imitación tecnológica de bienes de capital innovadores generados en el país foráneo. La premisa fundamental que subyace en el modelo es que en un entorno en donde existen países innovadores e imitadores de bienes de capital físico, la imitación tecnológica y la acumulación de capital físico para un país menos desarrollado se encuentran en la base del crecimiento económico. El planteamiento puede verse como una reflexión acerca de los posibles determinantes del crecimiento económico de un país menos desarrollado; la importancia de la propuesta radica en que es un esfuerzo analítico por colocar sobre el escritorio la discusión y la comprensión del crecimiento de estas economías.

Es conveniente insistir en que el modelo propuesto asume que el país desarrollado es uno de ingreso alto dedicado a la innovación tecnológica de bienes de capital, en tanto que el país menos desarrollado es uno de ingreso medio, como Brasil, México, Corea del Sur, Malasia y Sri Lanka, los cuales tienen la capacidad de aprender y adaptar los diseños innovadores generados fuera del espacio nacional. Se trata de países cuyas firmas pueden invertir en actividades de imitación de bienes de capital innovadores, dominar los procesos involucrados en el diseño innovador y adaptarlos a las necesidades del país local.

El trabajo se organiza como sigue: en la sección I se exponen las ideas básicas que versan sobre el crecimiento endógeno en un país menos desarrollado acotadas a las metodologías de Grossman y Helpman (1990) y Romer y RiveraBatiz (1991). En la sección II se expone la estructura productiva y la tasa de crecimiento del país innovador. En la sección III se analizan las características y la tasa de crecimiento del país imitador. En la sección IV se discuten los resultados. Y, por último, se presentan las conclusiones. 
12 ECONOMÍA: TEORÍA Y PRÁCTICA • Nueva Época, número 28, enero-junio 2008 


\section{IDEAS BÁSICAS DE CRECIMIENTO ENDÓGENO EN UN PAÍS MENOS DESARROLLADO}

Después de Solow (1956), la relación entre el comercio internacional y el crecimiento económico se estudió retomando la hipótesis de convergencia. Bajo el supuesto de que los países tienen parámetros estructurales similares, se insistía en que un país con menor dotación de capital físico por persona presentaría en el corto plazo tasas de crecimiento relativamente altas y en el largo plazo tendería a un estado de crecimiento equilibrado común al de los países con capital físico por persona alto.

En este marco se intuye que el progreso técnico fluye libremente de los países desarrollados a los países menos desarrollados. En un entorno de economías abiertas se puede afirmar que el comercio internacional facilita la difusión del progreso técnico y promueve el crecimiento económico; el inconveniente es que el progreso técnico es exógeno.

Al interior de la nueva bibliografía el análisis de esta relación ha seguido dos líneas de investigación. Primero, propuestas de economía abierta en donde el crecimiento económico es el resultado de un proceso de acumulación de capital físico a partir de learning-by-doing acompañado de externalidades, cuyas fuentes de inspiración son Romer (1986) y Lucas (1988), así como los trabajos de Young (1991), Lucas (1993) y Grenier (2003). Segundo, modelos en donde el crecimiento económico responde a actividades intencionales de investigación y desarrollo, como las propuestas de Romer (1987, 1990), Grossman y Helpman (1989a, 1989b, $1990,1991)$ y Aghion y Howitt $(1992,1998)$. Una generalidad de ambas metodologías es que el análisis se centra en los efectos del comercio internacional sobre el crecimiento económico de países desarrollados.

En las propuestas para países desarrollados se parte del supuesto de que la actividad de innovación y el capital físico son las fuerzas que explican el crecimiento económico de estos países. En las formalizaciones de crecimiento económico endógeno para países menos desarrollados (del tipo de Grossman y Helpman (1991) y Tirado (1995)) se parte del supuesto de que la capacidad de creación, absorción e imitación de la tecnología y la acumulación de capital físico son los responsables del crecimiento de estos países. Desde este punto de vista, las propuestas para países menos desarrollados pueden verse como una adaptación de los modelos de base de países desarrollados. En particular, basta sustituir grosso modo la especificación de la función de producción de diseños innovadores por la de diseños imitados. 
Los modelos de economía abierta más representativos para el análisis del crecimiento económico endógeno de un país desarrollado son de Grossman y Helpman (1990), Romer y Rivera-Batiz (1991) y Aghion y Howitt (1998). En términos del modelo ahora propuesto, a continuación se recuperan las ideas centrales de las metodologías de base más representativas de la segunda línea de investigación, se rescatan las ideas de Grossman y Helpman (1989b) y Romer (1990), y como extensiones para una economía abierta se retoma a Grossman y Helpman (1990) y Romer y Rivera-Batiz (1991). Y sólo entonces se procede a la inspección de las propuestas de Grossman y Helpman (1991) y Tirado (1995) como aproximaciones para el análisis del crecimiento económico de un país menos desarrollado.

Los modelos de base de Grossman y Helpman (1989 b) y Romer (1990) tienen la particularidad de que, en un entorno de tres sectores (el productor de bienes finales, el productor de diseños innovadores y el sector productor de bienes intermedios), la fuerza que impulsa el crecimiento económico es la innovación tecnológica a partir de la actividad de investigación y desarrollo. Los resultados sugieren que la tasa de crecimiento de la economía depende fundamentalmente de la mano de obra dedicada a la actividad de investigación y desarrollo. Esto responde, al menos parcialmente, a la pregunta de por qué las tasas de crecimiento entre países difiere; desde esta perspectiva, los países que tengan mayores montos de mano de obra en el sector de investigación y desarrollo tendrán tasas de crecimiento mayores, y viceversa. Estos resultados surgen de manera natural si se piensa que el sector de investigación y desarrollo tiene rendimientos crecientes a escala y que una mayor utilización de mano de obra implica mayores tasas de crecimiento en forma proporcional.

Uno de los puntos medulares en la bibliografía sobre crecimiento endógeno es la generación y transferencia de tecnología entre sectores. En los modelos de referencia la generación de conocimiento nuevo está a cargo del sector de diseños innovadores, comúnmente denominado como el sector de investigación y desarrollo. Tanto en Grossman y Helpman (1989b) como en Romer (1990) la producción de bienes finales utiliza como insumos los bienes intermedios; esta forma de operación otorga a los bienes intermedios el canal por el cual la tecnología se transfiere de un sector a otro.

Estas ideas de producción y utilización de los diseños innovadores adquieren mayor relevancia cuando se estudian en un entorno de economías abiertas. En lo que sigue se recogen las ideas básicas de Grossman y Helpman (1990) y Romer y Rivera-Batiz (1991) como extensiones de los modelos de base de economía cerrada. 
Grossman y Helpman (1990), con fundamento en Grossman y Helpman (1989b), en un marco de países desarrollados relaja el supuesto de economía cerrada al de una economía abierta con dos países y dos bienes finales. El resultado es que la tasa de crecimiento de cada país depende de la cantidad de mano de obra dedicada a la actividad de investigación y desarrollo, y de las ventajas comparativas generadas en la actividad de ID.

En el modelo cada país se dedica a tres actividades productivas: la producción de un bien final, la producción de variedades de insumos intermedios y la producción de diseños de insumos intermedios innovadores. Las características en cuanto a la producción y transferencia de la tecnología son similares al modelo de base. Por el lado de la demanda final, en ambos países se asume la presencia de un consumidor representativo con preferencias homotéticas cuyo problema es la maximización de la utilidad debido al consumo de bienes finales.

El productor de insumos intermedios de cada país, al tomar como dada la curva de demanda inversa que le impone el productor de bienes finales y los costos salariales debidos a la producción de los insumos, tiene el problema de elección del precio de oligopolio que le maximice el beneficio. Los consumidores de ambos países por su parte maximizan la utilidad sujetos a su restricción presupuestaria.

Al igual que en el modelo básico, para cada país se calculan la condiciones de no arbitraje de los beneficios del sector productor de insumos intermedios, se deducen las ecuaciones de movimiento del gasto de los consumidores y la condición de no arbitraje de mercado de trabajo. Posteriormente, se encuentra la condición de equilibrio estático del mercado mundial de bienes finales y los precios relativos de cada bien final. Bajo el supuesto de que la fuerza que mueve el crecimiento económico de cada país es la actividad de innovación tecnológica, se tiene la siguiente tasa de crecimiento:

$$
g=H-\frac{\beta e}{\sigma}-\frac{1-\beta}{\alpha} s e,
$$

donde $i=1,2$ es el país; $H$ es la fuerza efectiva total; $\sigma \equiv \sum_{i} \sigma_{i} b_{i}$ es el promedio ponderado de los parámetros de ventaja comparativa $\left(b_{i}\right)$ con participación de producto como ponderador $\left(\sigma_{i}\right)$ y $s=\sum_{i} s_{i} / b_{i}$ con $s_{i}$ indicando la participación del gasto mundial asignado al bien final $Y_{i}$. En este modelo la tasa de crecimiento ( $g$ ) del país depende fundamentalmente de la fuerza efectiva total utilizada en los sectores de investigación y desarrollo y de la ventaja comparativa generada en dicho sector. 
Observe que el parámetro $\sigma$ ofrece un resumen de la asignación de recursos intersectoriales estático, que aumenta si la tasa de crecimiento del número de insumos intermedios diferenciados en el país con desventaja comparativa en investigación y desarrollo excede a la del otro país; si esta desventaja es mayor a la del otro país, el crecimiento económico del local decrecerá, y viceversa. Lo importante en Grossman y Helpman (1990) es el reconocimiento de que las diferencias de innovación tecnológica entre países dan lugar a ventajas comparativas en un marco en donde el crecimiento es endógeno, un hallazgo muy propio de la bibliografía sobre este tipo de crecimiento para economías abiertas.

La otra línea metodológica para el análisis del crecimiento endógeno de una economía abierta es la establecida por Romer y Rivera-Batiz (1991). Estos autores se proponen analizar los efectos de la integración económica en una situación en donde existen dos países desarrollados. Igual que en Romer (1990), cada país consta de tres sectores, a saber: el sector productor de bienes finales, el productor de diseños innovadores y de manera virtual el productor de bienes de capital. El productor de bienes finales demanda bienes de capital, el productor de bienes de capital demanda diseños innovadores, mientras que el sector de bienes finales simplemente los vende a las familias.

En cada país la incorporación de tecnología a la actividad económica se da a partir de la producción de diseños innovadores del sector de investigación y desarrollo. Este sector, según Romer y Rivera-Batiz (1991), puede utilizar una función de producción con rendimientos crecientes a escala, teniendo como insumos el capital humano y un acervo de conocimientos ya existentes, o bien puede utilizar una función de producción con rendimientos constantes a escala, considerando como insumos la mano de obra calificada, la mano de obra no calificada y un continuo de insumos intermedios. La especificación de la función de producción es decisiva a la hora de estudiar los efectos de la integración comercial sobre la tasa de crecimiento de los países.

En ambos países la transferencia de tecnología se da a partir de la demanda de insumos intermedios por parte del sector productor de bienes finales. En contraste con la metodología de Grossman y Helpman (1991), en donde aparece claramente el proceso de producción del bien intermedio, en la metodología de Romer y Rivera-Batiz (1991) se presentan las condiciones que garantizan la producción de una unidad adicional del bien de capital nuevo pero no queda explícita la producción del bien.

Afín a la metodología de Romer (1990), el comportamiento monopólico de los productores de diseños y de bienes de capital, al tener como incentivo la 
ganancia, crea las condiciones para seguir innovando y acumulando capital físico, y así juntos ser los responsables en gran parte del crecimiento económico de los países.

Ante este marco, Romer y Rivera-Batiz (1991) proponen tres simulaciones para descifrar los efectos de la integración comercial en dos países desarrollados. Los resultados son sorprendentes:

a) En el modelo de manejo de conocimiento del sector de investigación y desarrollo, con flujo de bienes y sin flujo de ideas, sustentado en la función de producción $\dot{A}=\delta H_{A} A$, se concluye que, si bien es cierto que la integración comercial aumenta la productividad marginal del capital humano de ambos países y duplica el precio de las patentes, la tasa de crecimiento mundial no se ve afectada.

b) En la especificación manejo de conocimiento con flujo de bienes e ideas, sustentada también en la función de producción $\dot{A}=\delta H_{A} A$, la integración económica aumenta la tasa de crecimiento de largo plazo. Si se asume que únicamente hay flujo de ideas, los resultados sugieren que la tasa de crecimiento dependerá del traslape de ideas entre los dos países.

c) Por último, en la especificación equipo de laboratorio del sector de investigación y desarrollo, analizado a partir de la función de producción $\dot{A}=H^{\alpha} L^{\beta} \int_{0}^{A} x(i)^{1-\alpha-\beta} d i$, se deduce que la integración comercial tiene efectos positivos sobre la tasa de crecimiento mundial.

Los resultados avalan que los rendimientos crecientes a escala en la producción de diseños son los responsables de que la integración comercial ejerza efectos positivos en el crecimiento económico mundial. Recordemos que en la nueva bibliografía sobre crecimiento económico los rendimientos crecientes a escala surgen debido a los efectos del derrame de conocimiento nuevo entre sectores y entre países, lo que a su vez responde a actividades intencionadas de investigación y desarrollo (Coe y Helpman, 1995). Desde este punto de vista es importante la aceptación de redundancia en la actividad de investigación y desarrollo; la creación de diseños innovadores requiere de recursos como cualquier otra actividad económica.

Los planteamientos de crecimiento endógeno para un país menos desarrollado tienen como fundamento los modelos de base arriba mencionados, aunque con ligeras modificaciones dirigidas hacia la especificación del sector de diseños 
imitados y a la búsqueda de la trayectoria de crecimiento del país imitador. Las aproximaciones más representativas son las de Grossman y Helpman (1991) y Tirado (1995).

La línea metodológica establecida por Grossman y Helpman (1991) analiza una economía mundial con dos regiones: la región norte industrializada, dedicada a la innovación de una variedad de insumos intermedios, y la región sur de ingreso medio, en donde las firmas dedican recursos a las tareas de aprendizaje y adaptación de tecnologías que han sido desarrollados en el norte. Bajo este supuesto la actividad de imitación representa un medio alternativo de diseminación de los resultados de investigación y desarrollo.

La producción de diseños innovadores e imitados se da en un contexto de monopolio en donde el único insumo utilizado es la mano de obra. Esto refleja la propiedad de rendimientos creciente a escala en la actividad de investigación y desarrollo, y rendimientos no decrecientes al nivel del único factor utilizado, una característica muy propia de las propuestas del tipo de Romer (1990), Grossman y Helpman (1991), y Aghion y Howitt (1992). En la producción de insumos intermedios, una vez que se termina una innovación, el país menos desarrollado la adapta y emprende su fabricación. En un escenario de competencia oligopólica, cada uno de estos productores irá en busca de su ganancia máxima.

Después de establecer las condiciones de optimización en el problema de las familias, las condiciones de maximización de los productores y el flujo de ganancias del productor de insumos intermedios se arriba a la trayectoria de innovación e imitación. En este nivel el análisis cobra fuerza; se estudian las tasas de crecimiento de cada país, tanto en condiciones de autarquía como en condiciones de integración. La tasa de crecimiento del país innovador en condiciones de autarquía depende fundamentalmente de la cantidad de trabajo asignado al sector de investigación. En un sentido similar, la tasa de crecimiento del país imitador depende positivamente de la cantidad de mano de obra utilizada en el sector de diseños. Mientras que en condiciones de libre comercio la tasa de crecimiento del país imitador depende de la razón entre salarios del sur y salarios del norte.

Tirado (1995) retoma el marco de Romer y Rivera-Batiz (1991) para analizar la tasa de crecimiento de un país imitador menos desarrollado que guarda relación con un país desarrollado innovador a partir del mercado de tecnología mundial. En este esquema, cada país se conforma de dos sectores: el productor de bienes finales y el productor de diseños. El país innovador se caracteriza por generar permanentemente nuevos productos mediante la aplicación en la manu- 
factura de diseños innovadores creados en los laboratorios de investigación. El país imitador se caracteriza por producir bienes que fueron desarrollados originalmente en el país innovador pero que son rediseñados por el sector de investigación y desarrollo del país local.

En el país desarrollado el sector de bienes finales posee una tecnología de rendimientos constantes a escala y utiliza como insumos capital humano, capital físico y un acervo de diseños innovadores adquiridos del sector de investigación y desarrollo, mientras que el productor de diseños, igual que en Romer (1990) y Romer y Rivera-Batiz (1991), utiliza como insumos capital humano y un acervo de diseños industriales prexistente; se trata de una función de producción con rendimientos crecientes a escala. Al productor de bienes finales, al demandar diseños innovadores del sector de investigación y desarrollo, se le impone un precio de monopolio. Después del cálculo de las dotaciones de capital humano de equilibrio se llega a la siguiente tasa de crecimiento:

$$
g_{n}=\delta H_{n}-(\alpha / \beta)
$$

Conforme a la ecuación, la tasa de crecimiento del país innovador $\left(g_{n}\right)$ depende principalmente del capital humano existente en la economía $\left(H_{n}\right)$ y los parámetros propios del capital humano $(\alpha$ y $\beta)$.

En el país imitador, el productor de bienes finales que opera bajo competencia perfecta, utiliza como insumos capital humano, mano de obra no calificada y un acervo de diseños imitados del país innovador; en tanto que el productor de diseños imitados, bajo un comportamiento monopólico, utiliza como insumos capital humano y un acervo de diseños innovadores comprados al productor de diseños innovadores foráneo.

El productor de diseños imitados compra diseños nuevos al país innovador y los adapta a las condiciones específicas del mercado y a las necesidades del país local, para después venderlos al sector productor de bienes finales. En el nivel local, se considera que hay una forma de mercado intrafirma, donde los diseños imitados son vendidos al sector que produce bienes finales. En este modelo, a diferencia de Romer (1990) y Romer y Rivera-Batiz (1991), se define la innovación como la proliferación de nuevos productos, como la creación de novedades tecnológicas dentro del mercado mundial; esto es, los bienes son nuevos en la medida en que no existían en el mercado mundial; por otra parte, la imitación se define como la actividad de reproducción de bienes novedosos.

Después de que cada productor resuelve su problema de optimización, se 
encuentra la asignación de capital humano de equilibrio, y la tasa de crecimiento de la economía toma la siguiente forma:

$$
g_{m}=\delta_{m} H_{m}(A / M)-(\Omega / \rho),
$$

donde $\delta_{m}$ es el parámetro de productividad del sector de investigación y desarrollo, y $\Omega$ y $\rho$ son los parámetros del capital humano utilizado en ambos sectores, respectivamente. La ecuación describe que la tasa de crecimiento $\left(g_{m}\right)$ en condiciones imitativas depende tanto del capital humano total $\left(H_{m}\right)$ como de la razón entre los diseños innovadores y los diseños imitados $(A / M)$. Esto último puede interpretarse como la brecha tecnológica existente entre el país que produce diseños nuevos y el país que los adapta.

Es necesario insistir que acorde con la línea de investigación sugerida por Grossman y Helpman (1989b, 1991), Romer (1990) y Aghion y Howitt (1992), en Tirado (1995) se subraya el carácter de rendimientos crecientes a escala del sector de investigación y desarrollo y sus efectos sobre la trayectoria de crecimiento equilibrado. Sin embargo, se heredan los problemas metodológicos presentes en Romer (1990) y Romer y Rivera-Batiz (1991); por ejemplo, no se explicitan las condiciones de producción del bien de capital, además de que el capital físico es exógeno y no hay un tratamiento específico respecto al financiamiento de la actividad de innovación e imitación tecnológica. Aunque quizá, al compararse con Romer (1990) y Romer y Rivera- Batiz (1991), uno de los inconvenientes más graves es la eliminación del lado de la demanda final, cuestión que trata de corregirse en este documento.

\section{El PAÍS INNOVADOR}

En el contexto de Romer (1990), la característica central del país innovador es la de tener a su cargo la producción de diseños de bienes de capital innovadores, mismos que le vende al país imitador a partir del mercado internacional de tecnología. Esto es, se asume que en el país innovador existe un sector productor de diseños de bienes de capital nuevos que vende sus bienes al productor de diseños imitados del país local.

En este escrito, al igual que en Grossman y Helpman (1989b) y Romer (1990), se define la innovación tecnológica como la proliferación de nuevos bienes de capital que se lanzan al mercado internacional de tecnología por parte de un país innovador, entiendiendo por nuevo lo que en el mercado mundial no 
existía anteriormente. A diferencia de Solow (1956) y Swan (1956), aquí se sostiene que aun cuando el conocimiento sea un bien no competitivo, su precio y la característica de ser parcialmente excluible dificulta su tránsito entre los sectores y los países.

Más adelante se caracteriza la estructura económica del país innovador, acentuando la producción de los diseños de bienes de capital innovadores y su desempeño en el crecimiento económico de dicho país. Por el lado de la producción, el modelo está conformado por tres sectores: el productor de bienes finales, el productor de conocimiento nuevo y en forma virtual el sector productor de bienes de capital. Por el lado de la demanda final, se supone la existencia de un consumidor representativo del tipo de Ramsey-Cass- Koopmans.

\section{Productor de bienes finales}

El país innovador, $n$, posee un sector productor de bienes finales, el cual opera bajo competencia perfecta. La producción se lleva a cabo utilizando la siguiente función de producción:

$$
Q_{n}=H_{q n}^{\alpha} L_{n}^{\beta} \int_{0}^{A} x_{n}(i)^{1-\alpha-\beta} d i
$$

El sector produce bienes finales, $Q_{n}$, con una tecnología que presenta las condiciones usuales de rendimientos constantes a escala y decrecientes a los factores. Se emplea capital humano, $H_{q n}$, trabajo menos calificado, $L_{n}$, y un continuo de bienes de capital denominado con la letra $i$.

La nueva bibliografía del crecimiento económico reconoce la importancia del capital humano como insumo en la producción de conocimiento y de bienes finales. En los modelos del tipo de Romer (1990) y Rivera-Batiz (1991) se insiste en el efecto directo de esta variable en la producción de conocimiento nuevo.

El sentido en el que se incorpora el capital humano en las funciones de producción de las firmas es que éstas emplean habilidades intelectuales de personal calificado cuya formación es la suma de educación formal y el aprendizaje en el trabajo, tal como Lucas (1988 y 1993) y Romer (1986 y 1990) reconocen. En estas propuestas interesa un concepto de capital humano entendido como fuente creadora de conocimiento y productora de bienes, sin hacer referencia a aquella elaboración teórica vinculada con las actitudes educativas y de salud de los individuos, que llevan en última instancia a reflexiones sobre la conducta económica de las familias. 


\section{Productor de conocimientos}

El segundo sector del país innovador es el productor de diseños innovadores. La producción de conocimientos se realiza con una tecnología de rendimientos crecientes a escala y no decrecientes en los factores empleados; aquí subyace el supuesto de que la posibilidad de duplicar el conocimiento innovador requiere menos empleo de recursos en relación con la posibilidad de producir un conocimiento innovador. Formalmente, el flujo de diseños innovadores producidos en el sector de investigación se define mediante la siguiente ecuación:

$$
\dot{A}_{n}=\delta_{n} H_{a n} A_{n}
$$

donde $\delta_{n}$ es un parámetro de productividad. De acuerdo con la ecuación (2), para la producción de conocimiento se emplea capital humano, $H_{a n}$, y un acervo de conocimientos empleados en la producción de nuevos diseños, $A_{n}$, que se supone es un bien sin competencia para el productor.

La ecuación (2) describe que la variación en el tiempo de los diseños nuevos es una función positiva del capital humano que utiliza el sector de investigación y desarrollo denotado mediante $H_{a n}$, y es una función positiva del acervo de diseños ya existentes simbolizado por $A_{n}$. Un supuesto implícito en la función de producción es que la creación de diseños nuevos es ilimitada debido a que no hay límites a la capacidad humana para la generación de innovación tecnológica. Además, dado que el salario del capital humano utilizado en el sector crece en forma proporcional a $A$, se intuye que si crece $A$ el salario también lo hará, lo cual incentivará la utilización de $H_{a n}$.

\section{La interrelación entre los sectores}

El modelo plantea que el productor de bienes de capital le compra diseños al sector productor de diseños nuevos a un precio $P_{A}$; posteriormente, al generar sus bienes le vende al sector productor de bienes finales cobrando un precio de monopolio, mientras que el productor de bienes finales le vende simplemente sus bienes a los consumidores.

El productor de bienes finales, el cual opera bajo competencia perfecta, demanda bienes de capital. El problema del productor consiste en la elección de $x_{n}$ que le maximice el beneficio. Formalmente, 


$$
\begin{gathered}
\text { máx } \\
X_{N}
\end{gathered} \quad \int_{0}^{A}\left[H_{q n}^{\alpha} L_{n}^{\beta} x_{n}(i)^{1-\alpha-\beta}-p_{n}(i) x_{n}(i)\right] d i,
$$

donde $H_{q n}^{\alpha} L_{n}^{\beta} x_{n}(i)^{1-\alpha-\beta}$ es el ingreso por las ventas de sus bienes y $p_{n}(i) x_{n}(i)$ es el costo de los bienes de capital.

Las condiciones de primer orden del problema (3) permiten afirmar que el productor de bienes finales le presenta al productor de bienes de capital la siguiente curva de demanda inversa,

$$
p_{n}(i)=(1-\alpha-\beta) H_{q n}^{\alpha} L_{n}^{\beta} x_{n}(i)^{-\alpha-\beta} .
$$

La ecuación (4) es una condición para la compra y la venta del bien de capital; describe que el precio del bien debe ser igual a su producto marginal.

Por su parte, el productor de bienes de capital, al tomar como dada la ecuación (4), debe elegir la cantidad del bien de capital que le maximice la ganancia. Formalmente, el problema consiste en:

$$
\pi_{k n}={ }_{X_{N}}^{\text {máx }}\left[p_{n}(i) x_{n}-r_{n} x_{n}\right],
$$

donde el beneficio es igual al ingreso menos el costo, siendo el ingreso la ganancia del bien de capital al sector de bienes finales, denotado mediante $p_{n}(i) x_{n}$, y el costo es el interés $r_{n}$ sobre las unidades de producto detraídas, renunciando al consumo necesario para generar $x$ 's bienes de capital. De la solución del problema (5) surge el ingreso de monopolio $\bar{p}_{n}(i) \bar{x}_{n}(i)=(1-\alpha-\beta) H_{q n}^{\alpha} L_{n}^{\beta} x_{n}^{-1-\alpha-\beta}$ y el flujo de beneficios de monopolio:

$$
\pi_{k n}=(\alpha+\beta) \bar{p}_{n}(i) \bar{x}_{n}(i) .
$$

Ahora bien, sea $P_{A}$ el costo de un diseño nuevo que el productor de bienes de capital enfrenta. Considerando la condición de que el valor presente del flujo de beneficio descontado a la tasa de interés $r_{n}$ es igual al costo del diseño $P_{A}$, se tiene la relación:

$$
P_{A}=\frac{\pi_{k n}}{r_{n}}=\frac{(\alpha+\beta)}{r_{n}}(1-\alpha-\beta) H_{q n}^{\alpha} L_{n}^{\beta}{ }^{-1-\alpha-\beta} .
$$

Después de igualar los salarios del capital humano del sector productor de bienes finales y el sector productor de diseños (dados por $w_{H q^{n}}=\alpha H_{q n}^{\alpha-1} L_{n}^{\beta} A \bar{x}_{n}^{-1-\alpha-\beta}$ 
y $w_{\text {Han }}=\delta_{n} P_{A} A_{n}$, respectivamente), retomando $P_{x}$ se obtiene la cantidad óptima de capital humano utilizado en el sector de bienes finales,

$$
H_{q n}=\frac{\Lambda}{\delta_{n}} \mathrm{r}_{\mathrm{n}}, \text { donde } \Lambda=\frac{\alpha}{(\alpha+\beta)(1-\alpha-\beta)} .
$$

Del sector de investigación y desarrollo, si suponemos que las variables $Q_{n}$ y $K_{n}$ crecen a la tasa $g_{n}=\dot{A}_{n} / A_{n}$, se tiene que:

$$
g_{n}=\frac{\dot{Q_{n}}}{Q_{n}}=\frac{\dot{K}_{n}}{K_{n}}=\frac{\dot{A_{n}}}{A_{n}}=\delta_{n} H_{a n} .
$$

De la ecuación (7), considerando la condición market clearing en el mercado de capital humano, $H_{n}=H_{a n}+H_{q n}$, se llega a la tasa de crecimiento por el lado de la producción,

$$
g_{n}=\delta_{n} H_{n}-\Lambda r_{n} .
$$

El resultado es igual que en Romer (1990). La ecuación (8) describe que la tasa de crecimiento por el lado de la producción - la cual está guiada por la capacidad de innovación tecnológica - es una función creciente de la cantidad de capital humano total presente en la economía y decreciente de la tasa de interés. Esto último se debe a que el valor presente del flujo de beneficios monopólico tiende a disminuir en tanto $r_{n}$ tienda a aumentar.

\section{La tasa de crecimiento de la economía}

Siguiendo a Romer (1990), para el cierre del modelo se recupera la relación entre la tasa de crecimiento y la tasa de interés a partir de incorporar la demanda final en un contexto de Ramsey-Cass-Koopmans. El problema del consumidor consiste en maximizar la utilidad sujeta a la restricción de acumulación de capital físico.

Formalmente, el problema de maximización del consumidor,

$$
\underset{c}{\operatorname{Máx}} U_{n}(0)=\int_{0}^{\infty} U_{n}\left(C_{n}\right) e^{-\rho t} d t \text {, con } U_{n}^{\prime}(C)>0 \mathrm{y} U_{n}^{\prime \prime}(C)<0 \text {. }
$$

s. a:

$$
\dot{K}_{n}=w_{L n} L_{n}+w_{H n} H_{n}+r_{n} K_{n}-C_{n}, C_{n}(0) \geq 0 \mathrm{y} K_{n}(0) \geq 0 \text {. }
$$


La ecuación (9) describe el problema de maximización del consumidor del país innovador debido al consumo de bienes finales obtenidos del sector productor de bienes finales. El consumidor va a maximizar el flujo de utilidades instantáneas debido al consumo, considerando el factor $e^{-\rho t}$, en donde se asume que $\rho>0$, lo cual significa que las utilidades son menos valoradas entre más tarde son recibidas. Mientras que la ecuación (10) es la restricción de acumulación de la familia productora, expresa que la variación del capital físico aumenta con los ingresos percibidos por prestar sus servicios trabajo, calificado $\left(W_{L n}\right)$ y no calificado $\left(W_{H n}\right)$, en donde este último incluye el salario del consumidor bien sea por prestar sus servicios en el sector de investigación o en el sector de bienes finales; además, la variación del capital también aumenta debido a los ingresos percibidos por la renta de capital físico $(r K)$, y cae con el aumento del consumo $(C)$.

De las condiciones de primer orden de los problemas (9) y (10), se tiene la tasa de crecimiento del consumo. Para el caso de una función de utilidad $U\left(C_{n}\right)=1 n C_{n}$, se tiene la tasa de crecimiento de consumo óptima,

$$
\frac{\dot{C_{n}}}{C_{n}}=r_{n}-\rho .
$$

De la ecuación (11), haciendo que $\dot{C} / C=g_{n}$, despejando $r_{n}$ e insertando en la ecuación (8), se obtiene la tasa de crecimiento del país desarrollado,

$$
g_{n}=\frac{\dot{Y}}{Y}=\frac{\dot{K}}{K}=\frac{\dot{C}}{C}=\frac{\dot{A}}{A}=\frac{\delta_{n} H_{n}-\Lambda \rho}{(1+\Lambda)} .
$$

La ecuación (12) arroja algunas conclusiones sobre las causas por las cuales se puede explicar, al menos en una primera aproximación, el proceso de crecimiento económico de los países desarrollados. Las diferencias en tasas de crecimiento de largo plazo entre los países, según el resultado del modelo, sugieren que una causa esencial puede radicar en la capacidad de acumular capital humano, es decir, en las cualidades de los sistemas educativos para formar fuerza de trabajo calificada y, por la naturaleza de (12), de la capacidad de la industria para emplearla principalmente en actividades de producción de conocimientos.

Así, según la ecuación (12), la importancia del capital humano radica no sólo en su cualidad de insumo sino también como fuente creadora de conocimiento nuevo, el cual tiene un efecto directo sobre la tasa de crecimiento de las economías, tal como afirman Romer (1990) y Romer y Rivera-Batiz (1991). Aunque es conveniente señalar que en todos estos planteamientos el capital humano se toma como exógeno. 
El otro resultado es la relación decreciente entre el crecimiento económico y la tasa de descuento. Este resultado da cuenta de que entre más alta es la tasa de descuento, mayor es la preferencia por el consumo presente en contra del consumo futuro, lo cual implica un menor ahorro que afecta de manera adversa al crecimiento económico. Un parámetro $\rho$ que se hereda del modelo del tipo de Ramsey-Cass-Koopmans.

\section{EL PAÍS IMITADOR}

Al igual que el país innovador, $n$, el país imitador, $m$, se conforma de tres sectores: el sector productor de bienes finales, el productor de diseños imitados y en forma virtual el productor de bienes de capital imitados.

La característica principal del país imitador es que recurre al acervo de diseños innovadores para copiarlos y adaptarlos a la realidad específica del mercado y a las condiciones técnicas locales. En el modelo existe el supuesto de que el país menos desarrollado carece de condiciones para la innovación tecnológica y sólo puede copiar, adquirir patentes, adaptar diseños innovadores generados fuera del estado nacional (Tirado, 1995). En nuestra propuesta a la actividad de imitación se le ve más que como una desventaja como una oportunidad de los países menos desarrollados para alcanzar tasas de crecimiento positivas.

En el modelo se define la imitación tecnológica como la actividad de reproducir diseños de bienes de capital innovadores. Es conveniente mencionar que en la actividad de imitación, al igual que en la de innovación, la producción obedece a incentivos del mercado. Según este punto de vista, se reconoce que el conocimiento imitado posee un costo, es un bien sin competencia parcial, excluible, que requiere de asignación de recursos para su generación y apropiación.

\section{El productor de bienes finales}

Al igual que en Romer y Rivera-Batiz (1991), el país imitador $m$ cuenta con un sector productor de bienes finales que opera bajo competencia perfecta. Para la producción de bienes finales $\left(Q_{m}\right)$, la empresa productora utiliza como insumos trabajo calificado $\left(H_{q m}\right)$, mano de obra no calificada $\left(L_{q}\right)$ y un continuo $i$ de insumos intermedios importados. Formalmente:

$$
Q_{m}=H_{q m}^{\phi} L_{q}^{\psi} \int_{0}^{M} m_{m}(i)^{1-\phi-\psi} d i
$$


en donde el continuo $i$ de insumos intermedios importados que va desde cero hasta $M$ representa el acervo de bienes de capital imitados comprados al productor de dichos bienes. Se trata, por supuesto, de un número finito de insumos, aquellos que ya se han imitado y que están disponibles para usarse en cualquier momento. Evidentemente, dicha cantidad de insumos está determinada por el estado actual de imitación descrito mediante la letra $M$.

Una observación respecto a la estructura productiva del sector de bienes finales de ambos países es que en principio tanto el país innovador como el país imitador operan bajo mecanismos similares; por ejemplo, ambos demandan bienes de capital físico y capital humano, y ambos venden sus bienes finales a los consumidores. La diferencia está en la dotación y uso de los factores de la producción; aunque las funciones de producción de ambos sectores tengan la misma especificación funcional, en realidad son diferentes y las diferencias están dadas por los valores de los parámetros. Siguiendo a Mankiw (1995), debido a que las dotaciones de los factores y las intensidades en el uso de los factores son distintas, se puede afirmar que las funciones de producción son distintas.

\section{Productor de diseños imitados}

Retomando a Tirado (1995), el productor de diseños imitados utiliza como insumos capital humano $\left(H_{a m}\right)$ y diseños de bienes de capital innovadores $\left(A_{n}\right)$ generados en el país foráneo. La función de producción tiene la siguiente forma:

$$
\dot{M}_{m}=\delta_{m} H_{a m} A_{n} .
$$

La ecuación (14) describe que la cantidad de diseños a imitar es creciente en el tiempo de manera directamente proporcional a la cantidad de capital humano y al acervo de diseños innovadores a disposición del productor. Esta función de producción, al igual que la función de producción del productor de diseños del país innovador, posee rendimientos crecientes a escala y no decrecientes en los factores empleados. Detrás de esta afirmación subyace la idea de que la posibilidad de reproducir el conocimiento ya imitado requiere menos empleo de recursos que la inversión inicial, que implicó comprar la patente e imitarlo por primera vez.

En el modelo del país menos desarrollado descrito, el sentido en el cual se incorporan el capital humano y el conocimiento imitado es el mismo que en los planteamientos para el país desarrollado. El capital humano es un insumo y fuente creadora de conocimiento imitado, el acervo de diseños imitados se considera como un bien no competitivo y parcialmente excluible. Romer y Rivera-Batiz 
(1991) y Tirado $(1995,2003)$ incorporan estas características del capital humano en un contexto de comercio entre un país innovador y un país imitador. En algunos modelos de crecimiento endógeno para economías abiertas se incorpora el capital humano como determinante de la variedad de insumos que después ejerce efectos en la producción de bienes finales; por ejemplo, Grossman y Helpman (1991), en un marco de la relación norte-innovador y sur-imitador, establecen las condiciones para la innovación y la imitación tecnológicas, en donde el determinante principal es la cantidad de capital humano utilizado en los sectores productores de conocimiento.

\section{La interrelación entre los sectores}

En un esquema similar al de Romer (1990), el productor de bienes finales demanda bienes de capital imitados, mientras que el productor de diseños imitados compra diseños innovadores en el mercado internacional de tecnología a una empresa productora del país innovador. El productor de diseños imitados le vende sus bienes al productor de bienes de capital imitados a un precio $P_{m}$; este último le vende al sector de bienes finales, mientras que el productor de bienes finales ofrece sus bienes a los consumidores.

Así, el productor de bienes finales tiene que elegir la cantidad $m$ de insumos intermedios importados que le maximice el beneficio, dado su ingreso por la venta de sus bienes menos sus costos. Específicamente, se tiene el siguiente problema:

$$
\operatorname{máx}_{m_{m}} \int_{0}^{M}\left[H_{q m}^{\phi} L_{q}^{\psi} m_{m}(i)^{1-\phi-\psi}-p_{m}(i) m_{m}(i)\right] d i .
$$

De la condición de primer orden, se tiene que:

$$
p_{m}(i)=(1-\phi-\psi) H_{q m}^{\phi} L_{q}^{\psi} m_{m}(i)^{-\phi-\psi} .
$$

La ecuación (16) es la curva de demanda inversa que el productor de bienes finales le presenta al productor de bienes de capital.

Por su parte, el productor de bienes de capital, al tomar como dato la ecuación (16), tiene que elegir la cantidad de $m$ a vender que le maximice el beneficio; su problema consiste en:

$$
\pi_{k m}=\operatorname{máx}_{m_{m}}\left[p_{m}(i) m_{m}-r_{m} m_{m}\right] .
$$


De la condición de primer orden se tiene el precio de monopolio que fija el productor de bienes de capital imitados,

$$
p_{m}(i)=\frac{r_{m}}{(1-\phi-\psi)}(1-\phi-\psi) H_{q m}^{\phi} L_{q}^{\psi} \bar{m}_{m}^{1-\phi-\psi}
$$

De la ecuación anterior se define el flujo de ganancia monopólica obtenida por el productor de bienes de capital descrito mediante $\pi_{k m}=(\phi+\psi) \bar{p}_{m}(i) \bar{m}_{m}(i)$, donde $\bar{m}_{m}(i)$ es la cantidad de demanda implicada por la función de demanda inversa (16) y el precio de monopolio $\bar{p}_{m}(i)$.

La decisión de producir un nuevo bien de capital depende de la comparación de la corriente de ingresos netos descontada a la tasa $r_{m}$ y el costo de la inversión inicial en un diseño imitado dado por $P_{M}$. La condición que garantiza la producción de un bien de capital imitado adicional está dada por la ecuación

$$
P_{M}=\frac{(\phi+\psi)}{r_{m}}(1-\phi-\psi) H_{q m}^{\phi} L_{q}^{\psi} \bar{m}_{m}^{1-\phi-\psi} .
$$

Un aspecto central del modelo es la condición que guarda el país imitador de tecnología; el productor de diseños imitados, en la medida que es tomador de tecnología, también lo es de su precio. Como tomador de tecnología y del precio éste tiene que elegir la cantidad de $A_{n}$ que le maximice el beneficio. El problema de optimización parcial del productor de diseños imitados consiste en:

$$
\operatorname{máx}_{\mathrm{A}_{\mathrm{n}}}\left[\delta_{m} H_{a m} A_{n}-P_{a n} A_{n}\right] .
$$

La condición que permite el beneficio máximo está dada por la siguiente ecuación:

$$
P_{a n}=\delta_{m} H_{a m}
$$

La ecuación (20) expresa el nivel en que el productor de diseños innovadores fija el precio de su diseño foráneo, en tanto que el productor imitador fija la cantidad de capital humano a emplear en dicha actividad. De modo que el innovador y el imitador se relacionan en el entorno mundial únicamente a partir del mercado internacional de tecnología.

Siguiendo la lógica de la nueva bibliografía sobre crecimiento económico, el siguiente paso es hacer explícito el estado de crecimiento equilibrado del país menos desarrollado. Para encontrar la tasa de crecimiento equilibrado por el lado de la producción se debe encontrar el nivel de capital humano de equilibrio 
de cada sector, al cual $Q_{m}, K_{m}$ y $M_{m}$ crecerán a una tasa constante. La condición determinante para la asignación de capital humano entre el sector de bienes finales y el sector de diseños imitados es que los salarios sean equivalentes.

Los salarios son calculados a partir de los problemas de elección de las dotaciones óptimas de capital humano para cada sector. Para el productor de bienes finales, al fijar $\bar{m}_{m}$ se puede asumir que $\int_{0}^{M} \bar{m}(i)_{m}^{1-\phi-\psi}=M \bar{m}_{m}^{1-\phi-\psi}$, de modo que la función de producción toma la siguiente forma: $Q_{m}=H_{q m}^{\phi} L_{q m}^{\psi} M \bar{m}_{m}^{1-\phi-\psi}$. De este nuevo planteamiento de la función de producción, el productor tiene que elegir la cantidad de capital humano $H_{q m}$ a utilizar que le maximice el ingreso. La condición que garantiza un ingreso máximo, está dada por $W_{H q m}=\phi H_{q m}^{\phi-1} L_{q}^{\psi} M \bar{m}_{m}^{1-\phi-\psi}$, mientras que el productor de diseños imitados también tiene que elegir la cantidad $H_{a m}$ que le maximice el ingreso. La condición que garantiza un resultado óptimo está dada por la ecuación:

$$
W_{\text {Ham }}=\delta_{m} A_{n} P_{M} .
$$

Después de igualar ambos salarios y considerar el valor de $P_{M}$ se obtiene la cantidad de capital humano dedicado a la producción de bienes finales:

$$
H_{q m}=\frac{\Omega r_{m} M_{m}}{A_{n}}
$$

donde:

$$
\Omega=\frac{\phi}{(\phi+\psi)(1-\phi-\psi)}
$$

La ecuación (21) describe que en la medida que la cantidad de diseños innovadores aumente y la disposición a imitar aumente, la cantidad de capital humano para destinar al sector manufacturero se incrementará. Esta razón es similar al hallazgo de Tirado (1995), según el cual dicha razón puede tomar dos valores extremos: por un lado, el valor de uno, lo cual implicaría que toda la innovación es transferida al país imitador, dando lugar a que dependa exclusivamente de $\Omega$, $r_{m}$ y $\delta_{m}$, con una estructura similar a la del país innovador; por otro lado, si la razón es cercana a cero, implicaría una asignación muy baja de capital humano al sector manufacturero, dando lugar a la producción de bienes finales intensivos en mano de obra no calificada y/o en capital físico. 
Para encontrar la tasa de crecimiento equilibrado basta el supuesto de que la fuerza que impulsa el crecimiento económico en el país menos desarrollado es la imitación tecnológica de diseños de bienes de capital innovadores. Después de considerar la condición market clearing en el mercado de capital humano, $H_{m}=H_{a m}+H_{q m}$, se llega a la tasa de crecimiento por el lado de la producción:

$$
g_{m}=\frac{\dot{Q_{m}}}{Q_{m}}=\frac{\dot{K_{m}}}{K_{m}}=\frac{\dot{M}_{m}}{M_{m}}=\frac{\delta_{m} H_{m} A_{n}}{M_{m}}-\Omega r_{m}=\delta_{m} H_{m} B-\Omega r_{m},
$$

donde $B=A_{n} / M_{m}$. De la ecuación (22) se desprende que la tasa de crecimiento en condiciones imitativas depende tanto del capital humano total como de la razón entre diseños innovadores y diseños imitados. Conforme a Tirado (1995), esto último puede interpretarse como la brecha tecnológica entre el país que produce diseños nuevos y el país que las adapta.

Así, de la ecuación (22) surgen dos conclusiones de importancia. Primero, si todo lo demás es constante, ante un aumento en la cantidad de capital humano total en un momento dado, mayor también la fracción de tiempo que se dedicará a la actividad de imitación y mayor será la tasa de crecimiento. Segundo, lo demás es constante si la razón $B$ aumenta debido a que las innovaciones foráneas crecen más rápido, la asignación de capital humano a tareas imitativas aumenta, como la tasa de crecimiento.

\section{La tasa de crecimiento de la economía}

De acuerdo con la metodología de Romer (1990), para especificar la tasa de crecimiento de la economía del país imitador en el mismo sentido que en el país innovador, se inserta el lado de la demanda final en un contexto de Ramsey-CassKoopmans. El problema del consumidor representativo consiste en:

S. a:

$$
\operatorname{Max} U_{m}(0)=\int_{0}^{\infty} U\left(C_{m}\right) e^{-\varphi t} d t \text {, con } U_{m}{ }^{\prime}\left(C_{m}\right)>0 \text { y } U_{m}{ }^{\prime \prime}\left(C_{m}\right)<0
$$

$$
\dot{K}_{m}=w_{L m} L_{m}+w_{H m} H_{m}+r_{m} K_{m}-C_{m}, C_{m}(0) \geq 0 \text { y } K_{m}(0) \geq 0 .
$$

Las ecuaciones (23) y (24) conforman el problema del consumidor representativo del país imitador. La ecuación (23) describe la maximización de la utilidad del consumidor como un flujo de utilidades instantáneas que va desde 
cero hasta el infinito debido al consumo de bienes finales ponderado por la tasa de descuento. Mientras que la ecuación (24) es la variación del capital físico, al igual que en el caso del consumidor del país innovador, indica que éste aumenta con el ingreso, obtenido a partir de los salarios por los servicios trabajo $\left(W_{L m} \mathrm{y}\right.$ $\left.W_{H m}\right)$ y los ingresos percibidos por la renta del capital físico $\left(r_{m} K_{m}\right)$, y cae con el aumento del consumo $\left(C_{m}\right)$.

Si bien es cierto que las preferencias entre países son distintas debido a que varían los niveles de ingreso, los hábitos de consumo, los aspectos culturales y la estructura productiva, aquí se asume que aunque ambos países, innovador e imitador, tienen una estructura de preferencias similar con un mismo perfil de consumo, lo que los diferencia es la intensidad en el consumo de los bienes finales (Tugores, 2002).

Después de plantear la función hamiltoniana de valor presente y considerar las condiciones de primer orden, se encuentra la tasa de crecimiento del consumo. La tasa de crecimiento del consumo para el caso de una función de utilidad logarítmica, $U\left(C_{m}\right)=1 n C_{m}$, toma la siguiente forma:

$$
\frac{\dot{C_{m}}}{C_{m}}=r_{m}-\varphi .
$$

De la ecuación (25), haciendo $\dot{C}_{m} / C_{m}=g_{m}$, posteriormente despejando $r_{m}$ e insertando dicha expresión en la ecuación (22), se arriba a la tasa de crecimiento de la economía del país imitador,

$$
g_{m}=\delta_{m} H_{m} B-\Omega r_{m}=\frac{\delta_{m} H_{m} B-\Omega \varphi}{(1+\Omega)},
$$

donde $B=A_{n} / M_{m}$. De la ecuación (23) sobresalen dos resultados interesantes para la reflexión teórica acerca de los posibles determinantes del crecimiento económico en un país menos desarrollado.

El resultado de mayor importancia es la relación positiva entre el crecimiento económico del país imitador y la brecha tecnológica. La relación positiva entre ambas variables sugiere que, en la medida que la actividad de innovación aumente, también lo hará la actividad de imitación, aumentando así la cantidad de capital humano dedicada al sector de imitación de conocimiento. Este resultado relaciona varios aspectos del crecimiento económico de un país imitador, tales como las condiciones para la imitación, la transferencia de tecnología, la brecha tecnológica y el catching-up entre un país imitador y uno innovador, temas de gran discusión que más adelante se comentan de manera muy general. 
La otra relación de importancia es entre el crecimiento económico y el capital humano total de la economía. El crecimiento económico es una función positiva del capital humano existente en la economía, es un resultado muy característico de la bibliografía sobre crecimiento endógeno en propuestas del tipo de Romer (1990) y Romer y Rivera-Batiz (1991). Lo interesante del modelo es que aquí se rescata dicha relación para el caso de un país menos desarrollado. Para el país imitador se muestra que uno de los determinantes esenciales del crecimiento económico es la acumulación de capital humano, esto es, que una de las causas del crecimiento económico de estos países se debe a las cualidades de la mano de obra y de la capacidad de la industria para absorberla fundamentalmente en las actividades de imitación tecnológica.

Otro de los resultados no menos importante es la relación decreciente entre el crecimiento económico y la tasa de descuento, un resultado característico de los modelos del tipo de Ramsey-Cass-Koopmans. El resultado sugiere que entre más alta es la tasa de descuento mayor es la preferencia por el consumo presente en contra del consumo futuro. La relación entre la tasa de crecimiento y la tasa de descuento adquiere mayor relevancia analítica cuando se vincula con el ahorro en un contexto de intermediación financiera, permitiendo dilucidar la asignación de recursos hacia el crecimiento económico.

\section{Evaluación de los RESUltados}

El resultado central del modelo descrito en la ecuación (23) es la relación entre el crecimiento económico del país imitador, el capital humano y la brecha tecnológica. Es una manera bastante simplificada de establecer algunas de las condiciones bajo las cuales un país menos desarrollado puede imitar y alcanzar tasas de crecimiento positivas. Veamos la relevancia de las variables implicadas en este resultado.

\section{El capital humano y el crecimiento económico}

Según la ecuaciones (12) y (23), en ambos países la tasa de crecimiento es proporcional a la cantidad de capital humano existente en la economía. El resultado sugiere que con mayor capital humano se puede tener un crecimiento ilimitado en cada país, lo cual implica que las diferencias en las tasas de crecimiento entre países obedecen en parte a la dotación de capital humano, una característica pro- 
pia de los modelos de crecimiento endógeno del tipo de Romer (1990) y RiveraBatiz (1991).

La forma en que se ha incorporado el capital humano a la teoría del crecimiento tiene que ver con las aportaciones de dos autores: en primer lugar, Nelson y Phelps (1977), y después, ya en los terrenos de la nueva bibliografía de crecimiento está la propuesta de Lucas (1988 y 1993).

Nelson y Phelps (1966) definen el capital humano como el aumento en la calificación individual adquirida a partir de la educación formal que permite aumentar la capacidad individual para innovar y adaptar las nuevas tecnologías, punto de vista según el cual el capital humano es el medio por el que se acelera la difusión tecnológica entre las economías. Para Nelson y Phelps (1966), el nivel de escolaridad de la población y, en particular, el número de investigadores, son factores importantes para el crecimiento de un país, aunque recientemente autores como Conolly (2001) reconocen que la calidad de los investigadores también cuenta y su aportación a la productividad total y al crecimiento económico es determinante.

Por su parte, Lucas (1988) define el capital humano individual como el nivel de calificación general. Para este autor, las fuentes de acumulación del capital humano son dos. Primero, la educación, que se adquiere en la escuela a partir de la educación formal, y, segundo, el aprendizaje por la práctica (learning by doing), mediante la cual se incorporan conocimientos nuevos. En el modelo de Lucas (1988) se manifiesta que el capital humano acumulado en el tiempo repercute en la productividad total y por lo tanto en el crecimiento económico; también se señala que un nivel bajo de capital humano y capital físico puede mantener a un país rezagado económicamente.

En un artículo posterior, Lucas (1993) señala que uno de los problemas del crecimiento económico es el aprendizaje para asimilar las tecnologías importadas. Según este autor, la base de cualquier transferencia tecnológica está en la capacidad para iniciar un aprendizaje que permita dominar las tecnologías importadas. Al hacer un balance acerca de las experiencias de crecimiento de algunos países emergentes del sudeste asiático, insiste en que el crecimiento económico exitoso de estos países se debe fundamentalmente a que han iniciado un esfuerzo enorme de aprendizaje.

Desde estos puntos de vista y el modelo propuesto, el capital humano es una variable que debe figurar en la explicación del crecimiento de los países menos desarrollados. Una crítica al modelo propuesto, al igual que en Grossman y Helpman (1989b), Romer (1990) y Aghion y Howitt (1992), es la implicación 
de que a mayor asignación de mano de obra al sector de investigación y desarrollo el crecimiento económico se vuelve ilimitado; la evidencia empírica sugiere que dicha proporcionalidad no es exagerada (Tirado, 2003), aunque es cierto que algunos países, por ejemplo, los Estados Unidos y Japón, que tienen mayores recursos humanos en las actividades de investigación y desarrollo, poseen tasas de crecimiento positivas altas (Mowery y Rosemberg, 1989).

\section{La brecha tecnológica y el crecimiento económico}

En el modelo del país menos desarrollado, de la ecuación (23), manteniendo todo lo demás constante, si la razón entre diseños innovadores y diseños imitados aumenta - debido a que las innovaciones foráneas crecen más rápido- se tiene que la asignación de capital humano a tareas imitativas aumenta, y así la tasa de crecimiento lo hace también. Esto implica que las posibilidades de crecimiento económico para una economía imitativa como la descrita dependen principalmente de las capacidades innovativas foráneas; si éstas disminuyen su dinámica, la tasa de crecimiento del país local tiende a disminuir en la medida que desciende la cantidad de capital humano a emplear en adaptaciones tecnológicas.

Al relacionar a los dos países, suponiendo que los coeficientes $\delta_{n}, \Lambda, \rho$ y $(1+\Lambda)$ coinciden con sus contrapartes $\delta_{m}, \Omega, \phi$ y $(1+\Omega)$, y considerando las razones $H(t)=H n(t) / H m(t)$ y $B(t)=A(t) / M(t)$, pueden existir trayectorias de crecimiento positivas o negativas. En particular, se pueden tener tres casos:

Caso 1: Si $H(t)<B(t)$, entonces a lo largo de un periodo determinado la tasa de crecimiento económico en el país imitador será mayor que en el país innovador. Esto es, si la relación entre capitales humanos es menor a la relación existente entre los diseños innovadores e imitados, habrá un espacio de crecimiento en donde el país imitador tenderá a aumentar sus capacidades adaptativas hasta que las dos razones tiendan a igualarse.

Caso 2: En el caso de que $H(t)>B(t)$, se puede esperar que la tasa de crecimiento de la economía en el país innovador sea mayor a la existente en el país imitador en un momento dado. Es decir, en el caso en que el país innovador asigne cada vez más capital humano a los sectores productivos, se espera que la economía crezca en forma proporcional al aumento de dicho acervo acelerando la producción de bienes innovadores. Manteniendo todo lo demás constante, en estas circunstancias se espera que la brecha sea cada vez más amplia. 
Caso 3: Si $B(t)=1$, relacionando el caso 1, se puede decir que la brecha tecnológica se ha cerrado. En este punto se espera que las tasas de crecimiento se igualen aun cuando el acervo de capital humano difiera en cada una de las naciones. Este resultado expresa dos fenómenos de importancia para el crecimiento económico de los países menos desarrollados: primero, la cuestión de si, partiendo de tasas de crecimiento bajas, el país imitador puede alcanzar tasas de crecimiento similares a las del país desarrollado; y segundo, si partiendo de las condiciones de país imitador éste puede alcanzar las capacidades tecnológicas del país desarrollado. Ambas cuestiones tienen que ver con los fenómenos de la convergencia entre países y el catching-up, cuyas respuestas quedan abiertas para reflexiones posteriores.

Sin bien el asunto de la brecha tecnológica y el crecimiento económico de un país imitador es un tema sumamente complejo, los resultados arriba señalados apenas matizan algunos aspectos del tema. El análisis de la relación entre la brecha tecnológica y el crecimiento económico comienza con Vernon (1966), seguido por Krugman (1979) y Segerstrom (1991). Al interior de la teoría de crecimiento endógeno la discusión fue iniciada por Grossman y Helpman (1990 y 1991), Stockey (1990) y posteriormente seguida por Acemoglu y Zilibotti (2001) y Grossman y Steger (2007). En la nueva bibliografía, a partir del esquema de la relación norte-innovador y sur-imitador con variedad de insumos y firmas oligopólicas, se discuten las condiciones según las cuales se da la imitación tecnológica, la relación entre la brecha tecnológica y el crecimiento económico, y se discuten también las ventajas y desventajas del comercio internacional.

Grossman y Helpman (1991) se centran en las condiciones según las cuales puede darse la innovación del norte y la imitación del sur. Los autores muestran las posibilidades de trayectorias de innovación e imitación de steady-state estables y encuentran que la tasa de crecimiento de la innovación de las firmas del norte depende de las condiciones de imitación de las firmas del sur, principalmente de la asignación de mano de obra a las actividades de investigación y desarrollo.

Acemoglu y Zilliboti (2001) y Grossman y Steger (2007), en un contexto también de la relación norte-innovador y sur-imitador, incorporan los diferenciales de habilidades entre las firmas del norte y las del sur, señalan que la mano de obra no hábil de las firmas del sur puede ser un obstáculo para la transferencia 
de tecnología; de hecho, consideran que la mano de obra no hábil tiene escasa productividad y repercute escasamente en la tasa de crecimiento del producto.

Estas discusiones dejan en claro que uno de los determinantes de la brecha tecnológica y el crecimiento económico es la mano de obra utilizada en la producción de diseños innovadores o imitadores; en el fondo describen que la capacidad de creación y absorción de tecnologías novedosas depende del capital humano existente en las economías, hallazgos que también están presentes en los modelos de los países desarrollados y menos desarrollados descritos en este documento.

\section{La difusión de la tecnología entre sectores y países}

En el ámbito sectorial, en los dos países la transferencia de tecnología se lleva a cabo a partir de la compra y la venta de bienes de capital innovadores e imitados, respectivamente. Estos resultados dejan evidencia analítica de que la difusión de la tecnología no es libre, capturando la idea de que tanto la actividad de innovación como la de imitación obedecen a incentivos de mercado que independientemente de su no rivalidad son bienes parcialmente excluibles. Grossman y Helpman (1989a y 1989b) y Romer (1990) discuten las implicaciones de estas propiedades en el crecimiento económico: la no competencia favorece el crecimiento económico, mientras que la calidad de excluible puede obstruir el crecimiento económico. En los modelos descritos, las ecuaciones (18) y (20) representan los precios que fijan los productores de bienes de capital imitados y el costo del diseño innovador imitado, respectivamente, que son costos que cancelan la posibilidad de la libre difusión del conocimiento entre sectores.

Entre países, se observa también que el proceso de difusión es costoso. Recordemos que uno de los supuestos que subyace en el país menos desarrollado es que un país que no cuenta con las condiciones y capacidades para la innovación, pero que sí cuenta con las condiciones para copiar, adquirir patentes y adaptar diseños innovadores, recurre al mercado internacional de tecnología y sostiene relaciones comerciales con la firma de diseños innovadores del país externo. Según estos supuestos la tecnología no fluye de manera libre de un país a otro; la ecuación (20) describe el precio que el productor de diseños innovadores del país foráneo impone al productor de diseños imitados del país local. Desde este punto de vista se reconoce que el conocimiento imitado es un bien parcialmente excluible, incapaz de transitar libremente entre los países.

Sin embargo, es conveniente señalar que en la bibliografía sobre creci- 
miento endógeno se asume que el conocimiento innovador o imitador no puede fluir libremente, no es un resultado que se deduzca del modelo. En los modelos presentados también se toma este supuesto: el conocimiento innovador e imitador no pueden fluir libremente.

Otro aspecto de importancia en la nueva bibliografía sobre el crecimiento, a diferencia de la teoría del comercio convencional, es que se ha centrado en la transferencia de tecnología a partir de los insumos intermedios. Se subraya que la importación de bienes intermedios es el medio más directo de relacionar la transferencia de tecnología y el comercio con el crecimiento económico de un país menos desarrollado. Navaretti y Soloaga (2001) sostienen que los insumos más complejos importados desde países innovadores aumentan la productividad a niveles mayores que los insumos con tecnologías viejas.

En los modelos de crecimiento endógeno con variedad o calidad de insumos intermedios los argumentos se refuerzan; aparte de los efectos directos en la productividad total debido a su contenido tecnológico, la importación de insumos intermedios también aumenta el conjunto de tecnologías accesibles, posee efectos dinámicos a partir del learning by doing que impulsan la innovaciones secundarias y fomenta la ingeniería reversible y las compras a escala (Conolly, 2001).

A diferencia de Tirado (1995), quien insiste en la importación de bienes finales imitados, en el modelo del país menos desarrollado ahora descrito, a tono con Grossman y Helpman (1991), éstos acentúan la transferencia de tecnología a partir de la importación de diseños de bienes intermedios innovadores. El productor de bienes imitados compra diseños innovadores a una firma productora externa; después de imitar el diseño innovador del bien, el productor de diseños lo vende al sector productor de bienes finales, conformando así un círculo virtuoso para el crecimiento económico.

Evidentemente, todo este análisis es básico, pues el tema del crecimiento económico de un país imitador es muy complejo. En este sentido, el modelo presentado tan sólo expone un par de relaciones básicas que puede ayudar a entender el crecimiento económico de estas economías; el modelo sugiere que el capital humano, el acervo de conocimientos innovadores externo y el acervo de conocimiento imitado, deben de figurar en la explicación del crecimiento de los países de ingreso medio.

No hay que olvidar que la discusión puede ir más allá; por ejemplo, la incorporación del tema de las condiciones tecnológicas para la imitación puede ofrecer mayor amplitud explicativa a los temas del crecimiento de estos países. Una inspección hacia las ramas industriales deja ver la heterogeneidad en las 
capacidades tecnológicas de las industrias; no todas las industrias tienen las mismas capacidades tecnológicas (Zúñiga, Guzmán y Brown, 2007). En el mismo sentido, en el nivel de ramas industriales, se pueden tener resultados diversos; dependiendo de las condiciones tecnológicas del país imitador, en algunas industrias la compra de tecnologías externas podrá complementar la investigación y el desarrollo del país local, mientras en otros casos puede sustituirla implicando un mayor rezago y dependencia tecnológicas.

Pese a esta heterogeneidad en las capacidades tecnológicas, se pueden establecer estrategias óptimas de innovación y/o imitación tecnológicas (Zúñiga, Guzmán y Brown, 2007) a partir de las condiciones tecnológicas de cada rama. En el fondo, la elección de dichas estrategias dependerá basicamente de los costos de transacción de las tecnologías, evidentemente sujetas a incertidumbre, información asimétrica y riesgo moral (Williamson, 1985).

Estos aspectos dejan en claro que la dinámica de la innovación y/o imitación tecnológica de los países menos desarrollados puede ser diversa y compleja y demanda esquemas analíticos con tratamiento sectorial. Sin embargo, no se parte de nada, hay ideas y se tiene la evidencia empírica para reflexionar el tema en un entorno de cambio estructural (Tirado, 2003; Syrquin, 2003). Sin duda, ello implica el replanteamiento de los esquemas de crecimiento endógeno alejados del estado de crecimiento equilibrado.

\section{Conclusiones}

El modelo muestra que una forma plausible de generar crecimiento endógeno en un país menos desarrollado es por medio de la imitación tecnológica, entendiendo ésta como la actividad de reproducir diseños de bienes de capital nuevos generados en un país innovador. En términos de países, la actividad de imitación tecnológica se considera como una actividad exclusiva de determinados países de menor grado de desarrollo, que ante su incapacidad de innovación tienen la infraestructura, la maquinaria y el equipo y la mano de obra, necesarias para llevar a cabo las actividades de imitación. Aquí, se pueden ubicar países de mediano grado de desarrollo, denominados también emergentes, como México, Brasil y los países del sudeste asiático.

Bajo la premisa de que en los países menos desarrollados la fuerza que impulsa el crecimiento económico es la imitación tecnológica, los resultados sugieren que la tasa de crecimiento mantiene una relación creciente con la brecha tecnológica y el acervo de capital humano total de la economía, y como es carac- 
terístico en los modelos de crecimiento del tipo de Ramsey-Cass-Koopmans, la tasa de crecimiento es una función decreciente de la tasa de descuento.

La relación positiva de la tasa de crecimiento con la brecha tecnológica indica que en la medida en que la innovación tecnológica aumente, lo hará también la actividad de imitación. Es conveniente mencionar que en ambas actividades existe un incentivo para invertir recursos establecida por la búsqueda de ganancias. Mientras que la relación positiva con el capital humano - propia de los modelos de crecimiento endógeno del tipo de Romer (1990) - establece que otra posible causa del crecimiento económico de estos países se encuentra en la acumulación de capital humano dedicado a la actividad de imitación tecnológica, en el fondo la capacidad de acumulación de capital humano depende del sistema educativo y de las cualidades del sector industrial para absorberla y utilizarla en actividades de imitación tecnológica.

Es conveniente insistir en que los resultados logrados se basan en el supuesto de que el país menos desarrollado no tiene capacidad de generar bienes de capital nuevos y sólo puede comprar patentes en el mercado de tecnología internacional y adaptarlos a las necesidades del país local. Desde este punto de vista, la actividad de imitación tecnológica más que un defecto es una oportunidad de los países menos desarrollados para alcanzar tasas de crecimiento positivas. Evidentemente, como toda actividad económica, la actividad de imitación, teniendo como incentivo la ganancia, requiere de asignación de recursos y en este sentido los países que no asignen recursos hacia actividades de imitación difícilmente podrán alcanzar tasas de crecimiento positivas.

Los resultados arriba expuestos son comparables a los logrados por Tirado (1995), autor que propone un modelo de dos sectores: uno productor de bienes finales y otro imitador de diseños de bienes finales nuevos generados fuera del país. El resultado central del modelo es que la tasa de crecimiento de un país imitador es una función creciente de la brecha tecnológica y del capital humano dedicado a actividades de imitación.

Sin embargo, al compararse con el planteamiento de Tirado (1995), el modelo propuesto es distinto en tres aspectos: primero, se logra crecimiento endógeno a partir del supuesto de que la fuerza que mueve el crecimiento económico es la imitación tecnológica de bienes de capital nuevos generados fuera del espacio nacional, mientras que en el modelo citado se logra crecimiento endógeno a partir de la imitación tecnológica de bienes finales nuevos; segundo, la tasa de crecimiento del país imitador es una función positiva del capital humano total, al tiempo que en el modelo comentado es una función positiva del capital humano 
dedicado exclusivamente al sector de imitación; y tercero, la propuesta de Tirado (1995) se basa en la imitación tecnológica de bienes finales, y el problema es que no hay una especificación acerca del consumo de los bienes finales, esto es, se pierde el lado de la demanda final, en tanto que el modelo de imitación tecnológica expuesto tiene la ventaja de recuperar la demanda final.

Si bien las relaciones encontradas entre la brecha tecnológica, el capital humano y el crecimiento económico no son resultados que aclaren por completo el fenómeno del crecimiento de los países menos desarrollados, la propuesta puede verse como una reflexión acerca del crecimiento económico de estos países; se trata de un resultado parcial que pone en la mesa la discusión de que la imitación tecnológica, la brecha tecnológica y el capital humano deben de figurar como causas de un fenómeno tan complejo como lo es el crecimiento económico de los países de ingreso medio.

\section{REFERENCIAS BIBLIOGRÁFICAS}

Acemoglu, D., y F. Zilibotti (2001), "Productivity differences", Quarterly Journal of 60 Economics, 116, pp. 563-606.

Aghion, Philipe, y Meter Howitt (1992), "A Model of Growth Trohough Creative Destruction”, Econometrica, 60, pp. 323-351. (1998), Endogenows Growth Theory, Cambridge Massachusetts, мIт Press.

Barro, Robert, y Xavier Sala-I-Martin (2003), Economic Growth, McGraw-Hill, Nueva York.

Coe, D., y E. Helpman (1995), "International R\&D Spillovers", European Economic Review, núm. 39: 859-887.

Conolly, Michelle (2001), “The Dual Nature of Trade: Measuring its Impact on Imitation and Growth", Working Paper, Duke University, núms. 97-34.

Eaton, Jonathan, y Samuel Kortum (2001), "Technology, Trade, and Growth: A Unified Framework”, European Economic Review, núm. 45, pp 742-755.

Grenier, Alfred (2003), "On the Dynamics of an Endogenous Growth Model with Learning by Doing", Economic Theory, vol. 21, pp. 205-214.

Grossman, Gene, y Elhanan Helpman (1989a), "Endogenous Products Cycles", Working Papers, núm. 2913, Oficina Nacional de Investigación Económica.

- y- (1989b), "Groath and Welfare in a Small Open Economy", Working Papers, núm. 2970.

Economic Review, vol. 80, núm. 4, pp. 796-815. 
Grossman, Gene y__ (1991), Innovation and Growth in the Global Economy, Cambridge Massachusetts, MIT Press.

Grossman, Volker, y Thomas M. Steger (2007), "Growth, Development and Tecnological Change", Discussion Papers Series, IZA DP 2558.

Krugman, Paul R. (1979), “A Model of Innovation, Technology Transfer, and the World Distribution Income”, Journal of Political Economy, 87, pp. 253-266.

Lucas, Robert E. (1988), “On the Mechanics of Economic Development”, Journal of Monetary Economics, 22, pp. 3-42. (1993), "Making a Miracle", Econometrica, 61.

Mankiw, N. G. (1995), “The Growth of Nations”, Brooking Papers on Economic Activity, 25, pp. 275-310.

Moweery, David, y Natham Rosemberg (1989), Technology and the Pursuit of Economic Growth, Cambridge University Press, Cambridge.

Navaretti, Giorgio Barba, e Isidro Soloaga (2001), "Weightless Machines and Costless Knowledge: An Empirical Analysis of Trade and Technology Diffusion”, Worldbank Paper, Núm. 2598.

Nelson, Richard R., y Edmund S. Phelps (1966), "Investment in Humans, Technological Diffusion, and Economic Growth", American Economic Review: Papers and Proceedings, 56 (1), pp. 69-75.

Romer, P. (1990), "Endogenous Technological Change”, Journal of Political Economy, 98, pp. 71-102.

— y Rivera-Batiz (1991), "Economic Integration and Endogenous Growth", Quarterly Journal of Economics, 106, pp. 531-555.

Segerstrom, P. S. (1991), "Innovation, Imitation and Economic Growth", Journal of Political Economy, vol. 29, núm. 4, pp. 807-827.

Solow, Robert M. (1956), "A Contribution to the Theory of Economic Growth", Quarterly Journal of Economics, 70, pp. 65-96.

Swan, Trevor W. (1956), "Economic Growth and Capital Accumulation”, Economic Record, 32, pp. 334-361.

Syrquin, Moshe (1994), "Structural Transformation and Growth Theory", en Pasinetti, L., y R. Solow (comps.), Economic Growth and the Structure of Long-Term Development, Londres, Macmillan.

Tirado, Ramón (1995), "Un modelo de crecimiento endógeno e imitación tecnológica", Estudios Económicos, 10, pp. 195-219. (2003), "La nueva teoría del crecimiento y los países menos desarrollados", Comercio Exterior, vol. 53, núm. 10, pp. 918-934. 
Tugores, J. (2002), Economía internacional, Globalización e integración regional. McGraw-Hill, Madrid.

Vernon, Raymond (1966), "International Investment and International Trade in the Product Cycle", Quarterly Journal of Economics, 80 (2), 190-207.

Williamson, O. (1985), The Economic Institutions of Capitalism, Nueva York, Free Press.

Young, A. (1991), "Learning by Doing and the Dynamics Effects of International Trade", Quarterly Journal of Economics, 106, pp. 369-405.

Zúñiga, María, Alenka Guzmán y Flor Brown (2007), “Technology Acquisition Strategies in the Pharmaceutical Industry in México", Comparative Technology Transfer and Society, vol. 3, núm. 3, pp. 274-296. 